\title{
The Relationship between Recurrent Aphthous Stomatitis and Iron Deficiency Anemia
}

\author{
Nurdiana, Pocut Astari \\ Department of Oral Medicine \\ Faculty of Dentistry, Universitas Sumatera Utara \\ Medan, Indonesia \\ nurdiana@usu.ac.id
}

\begin{abstract}
Recurrent aphthous stomatitis (RAS) is a common oral mucosal disease characterized by regular, round or oval, painful ulcers with an erythematous border that recur on a regular basis on the nonkeratinized oral mucosae such as labial, buccal, alveolar, and ventral tongue mucosa. Different etiologies and mechanisms have been postulated, resulting in identification of a variety of predisposing factors. A variety of underlying disorders may predispose patients to develop RAS; they include iron deficiency anemia. Iron deficiency anemia (IDA) is defined as a reduction in total body iron to an extent that iron stores are fully exhausted and some more degree of tissue iron deficiency is present. The aim of this review is therefore to discuss the relationship between RAS and iron deficiency anemia. IDA should be suspected in every case of RAS when no other obvious causes are identified. These findings are believed to be cause by the impaired cellular immunity, deficient bactericidal activity of polymorphonuclear leukocytes, inadequate antibody response, and epithelial abnormalities attributed to iron lack. RAS is a multifactorial disease with one of the common suspected etiology is IDA. Hence routine hematological examination may be carried out in RAS patients.
\end{abstract}

Keywords-recurrent aphthous stomatitis, etiology, iron deficiency anemia

\section{INTRODUCTION}

Recurrent aphthous stomatitis (RAS) is one of the most common oral mucosal lesions that physicians and dentists encounter. RAS also known as recurrent aphthous ulcers or canker sores [1]. RAS is characterized by recurring ulcers confined to the oral mucosa in patients with no other signs of systemic disease. RAS occurs in $20 \%$ of the population and if the ethnic or socioeconomic group is specifically investigated the incidence is 5 to $50 \%$ [2]. RAS is characterized by recurrent, small, round, or ovoid ulcers often multiple with circumscribed margin, erythematous haloes, and yellow or grey floors that present first in the childhood or adolescence [3].

Clinically, RAS occur in three different clinical morphological variants with number and size of the ulcers are the two main criteria used to divide ulcers into three forms which is minor, major, and herpetiform [1,4]. Despite their distinct characteristics, all forms of RAS have a significant impact on quality of life and interfere with activities of daily living [5].
RAS is a disorder of unknown etiology that may cause significant morbidity [1]. Different etiologies and mechanisms have been postulated, resulting in identification of a variety of predisposing factors. Researchers are still trying to find the exact etiology of this painful condition [6]. A variety of underlying disorders may predispose patients to develop RAS; they include iron deficiency anemia, neutropenia, and folic acid or vitamin B12 deficiency, as well as a selective vitamin B12 resorption defect [4].

Through previous published literature correlation seems to be present between hematinic deficiencies and RAS [6]. Multiple nutritional deficiencies including deficiencies of vitamins B1, B2, B6, and B12, folate, iron, and ferritin are reported to be the possible etiologies of RAS [7]. Hematinic deficiencies are found in $20 \%$ of patients with RAS. Hematinic deficiency (Iron, Ferritin, Hematocrit, Folate and B12) have been reported to twice common in RAS patients as compared to controls [khan]. It has been suggested by the results of several studies that there is a significant role of hematological parameters in the pathogenesis of RAS, but there is still some controversy remains [6]. Hence the aim of this review is therefore to discuss the relationship between RAS and iron deficiency anemia.

\section{LITERATURE REVIEW}

\section{A. Recurrent apthous stomatitis}

RAS is a common oral mucosal disease characterized by regular, round or oval, painful ulcers with an erythematous border that recur on a regular basis on the nonkeratinized oral mucosae such as labial, buccal, alveolar, and ventral tongue mucosa $[8,9]$. RAS occur worldwide and are reported on every populated continent. The prevalence of RAS is $2-66 \%$ of the international population. In children and in some adult communities who are affected, the incidence of recurrent aphthous ulcer is higher in women and girls than in men or boys. Recurrent aphthous ulcer major has a typical onset after puberty and can persist for the remainder of an individual's life, although after late adulthood episodes become much less common [1].

Although several etiological factors have been proposed, the exact causes of RAS are still not very clear. Previous studies of tissue infiltrated mononuclear cells in RAS specimens favor the role of cell-mediated 
cytotoxicity in the immunopathogenesis of RAS [7]. The major factors presently linked to RAS include genetic factors, hematologic or immunologic abnormalities, and local factors, such as trauma and smoking [2]. Genetic factors may be important. Family history is positive in up to $40 \%$ of patients. No consistent association with an HLA haplotype has been shown [4]. Hematinic deficiency involving reduced levels of iron, folic acid, or vitamin B12 has been found in a minority of patients with RAS and correction has led to resolution of symptoms [8]. It was initially reported that there is negative correlation between RAS and history of smoking, and many clinicians have reported that RAS is exacerbated when patients stop smoking. The nicotine metabolites are believed to decrease levels of proinflamatory cytokines and increase anti-inflammatory cytokines. Other factors that have been reported associated with RAS include anxiety, periods of psychological stress, localized trauma to the mucosa, menstruation, upper respiratory infections, and food allergy [2]. However, in the majority of cases it is difficult to identify the exact cause of SAR [8].

RAS occur in three different clinical morphological variants [4]. Minor-type (Mikulicz) are less than $1 \mathrm{~cm}$ in diameter and heal spontaneously in 4-14 days (Figure 1A). They account for $>70 \%$ of all RAS [4,5]. Major-type (Sutton disease or periadenitis mucosa necrotica recurrens) are usually $1-3 \mathrm{~cm}$ in diameter, deeply indurated and can last for 10 days to 6 weeks or occasionally even longer (Figure 1B). They account for around $10 \%$ of RAS $[2,4]$. About $64 \%$ of Sutton ulcers heal with scarring [4]. Herpetiform ulcers are very small grouped lesions (Figure 1C). They account for around $10 \%$ of RAS, are extremely painful [4,5]. As many as 100 ulcers can be present; they may coalesce into larger erosive plagues and about $32 \%$ heal with scarring (Table I) [4].

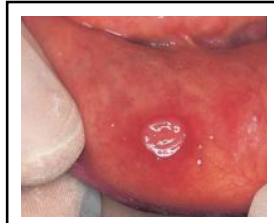

(A)

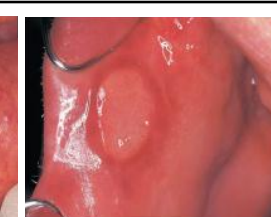

(B)

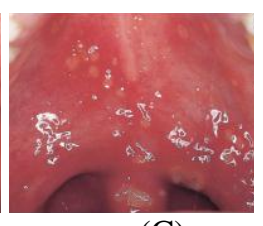

(C)
Figure 1. Minor-type (A), major-type (B), and herpetiform ulcers (C) of RAS [8]

The differential diagnosis includes a variety of diseases which can imitate the clinical picture of RAS [4]. Differential diagnoses of RAS include viral somatitis or erythema multiform [2].

Diagnosis of RAS is made relatively easily due to the characteristic clinical appearance of the ulcers and the recurrent nature of the symptoms. Biopsy is required in the major type SAR because ulcers in this type of SAR may resemble neoplasms or fungal infections [8].

Treatment for recurrent aphthous ulcers is directed at palliation of symptoms, shortening of healing time, and prophylaxis against future episodes [1]. No pharmacological treatment has been curative, although several modalities have been effective in decreasing pain and erythema and increasing the rate of reepithelialization associated with healing lesions [5]. The choice should be guided by disease severity, level of evidence, cost, and adverse effect profile [1]. It is reasonable to begin treatment with topical medication and advance to systemic medication and laser as necessary with a goal of decreasing recurrence rate and severity of the outbreaks [5]. Topical regimens may include topical corticosteroids (dexamethasone, triamcinolone, fluocinonide, and clobetasol), immunomodulatory agents (retinoid, cyclosporine, and amlexanox), antimicrobials (tetracycline, chlorhexidine gluconate, and dilute hydrogen peroxide), and anesthetics (topical lidocaine or benzocaine). Meanwhile, systemic agents may include systemic corticosteroids (prednisone and dexamethasone) and immunomodulatory agents (colchicine, azathioprine, and thalidomide) [1].

TABLE I. CLINICAL FEAURES OF MINOR, MAJOR, AND HERPETIFORM RAS [5

\begin{tabular}{|c|c|c|c|}
\hline & Minor & Major & Herpetiform \\
\hline $\begin{array}{l}\text { Gender } \\
\text { predilection }\end{array}$ & Equal & Equal & Female \\
\hline Morphology & $\begin{array}{c}\text { Round or oval } \\
\text { lesions } \\
\text { Gray-white } \\
\text { pseudo- } \\
\text { membrane } \\
\text { Erythematous } \\
\text { halo } \\
\end{array}$ & $\begin{array}{c}\text { Round or oval } \\
\text { lesions } \\
\text { Gray-white } \\
\text { pseudo- } \\
\text { membrane } \\
\text { Erythematous } \\
\text { halo } \\
\end{array}$ & $\begin{array}{c}\text { Small, deep } \\
\text { ulcers that } \\
\text { commonly } \\
\text { converge } \\
\text { Irregular } \\
\text { contour }\end{array}$ \\
\hline Distribution & $\begin{array}{l}\text { Lips, cheeks, } \\
\text { tongue, floor of } \\
\text { mouth }\end{array}$ & $\begin{array}{c}\text { Lips, soft } \\
\text { palate, pharynx }\end{array}$ & $\begin{array}{l}\text { Lips, cheeks, } \\
\text { tongue, floor } \\
\text { of mouth, } \\
\text { gingiva }\end{array}$ \\
\hline $\begin{array}{l}\text { Number of } \\
\text { ulcers }\end{array}$ & $1-5$ & $1-10$ & $10-100$ \\
\hline $\begin{array}{l}\text { Size of } \\
\text { ulcers }\end{array}$ & $<10 \mathrm{~mm}$ & $>10 \mathrm{~mm}$ & $2-3 \mathrm{~mm}$ \\
\hline Prognosis & $\begin{array}{l}\text { Lesions resolve } \\
\text { in } 4-14 \text { days } \\
\text { No scarring }\end{array}$ & $\begin{array}{c}\text { Lesions persist } \\
>6 \text { weeks } \\
\text { High risk of } \\
\text { scarring }\end{array}$ & $\begin{array}{l}\text { Lesions } \\
\text { resolve in } \\
<30 \text { days } \\
\text { Scarring } \\
\text { uncommon }\end{array}$ \\
\hline
\end{tabular}

\section{B. Iron deficiency anemia}

Iron deficiency anemia (IDA) is defined as a reduction in total body iron to an extent that iron stores are fully exhausted and some more degree of tissue iron deficiency is present [2]. IDA occurs when iron deficiency is severe enough to diminish erythropoiesis and cause the development of anemia [11]. Various metabolic processes require iron to function, including oxygen transport, DNA synthesis, and electron transport. Therefore, iron is very important for living organisms [10]. Iron deficiency causes microcytic anemia [7]. In practice, anemia is usually discovered and quantified by measurement of the RBC count, hemoglobin ( $\mathrm{Hb})$ concentration, and hematocrit [11].

Globally IDA is a serious health threat, resulting in an estimated 841,000 deaths [2]. IDA often occurs in women of childbearing age due to chronic blood loss 
associated with excessive menstruation. In adult males, IDA is usually associated with gastrointestinal diseases, such as peptic ulcer, diverticulosis, hiatal hernia, or malignancy [12]. Higher rates were also found in pregnant women and users of aspirin and/or antacids [10]. Other causes of IDA include inadequate intake of iron in the dietary and malabsorption of iron due to hypochlorhydria and diarrhea [13].

IDA patients may complain of weakness, tiredness (fatigue), palpitations, paresthesia and difficulty concentrating as well as brittle, flat, spoon-shaped nails (koilonychia) [12,13]. Patients also experience the sensation of tickle, distress, and impalement [13]. The initial oral manifestations of IDA are pale gingiva and labial mucosa. Other oral manifestations include atrophy of oral mucosa, angular cheilitis, ulceration, and redness, soreness or burning of tongue (atrophic glossitis) $[12,13]$.

Diagnosis of IDA can be based on clinical diagnosis such as koilonychia, neuropathy, angular cheilitis, and depapillation of tongue. Meanwhile, laboratory diagnosis may yield microcytic and hypochromic and peripheral smear show abnormal forms of RBCs. There is a reduced $\mathrm{Hb}$ level, as low as $4 \mathrm{~g} / 100 \mathrm{~mL}$. There is normal or slightly reduced RBC count. Mean corpuscular volume (MCV), mean corpuscular hemoglobin $(\mathrm{MCH})$ and mean corpuscular hemoglobin concentration (MCHC) are all reduced [13]. A definitive diagnosis of IDA requires evidence that iron stores are full depleted and is usually based on the finding of low serum iron, low transferrin saturation, and low ferritin. The most accurate initial diagnostic test for IDA is the serum ferritin level [2].

Iron deficiency should be treated with oral iron therapy in most patients, meanwhile the underlying etiology should be corrected so the deficiency does not recur [10]. Almost all patients with IDA can be treated by oral supplements of iron by giving ferrous fumarate or ferrous sulphate $[13,14]$. This supplement is given at a dose of $300 \mathrm{mg}$ three to four times per day for 6 months [14].

\section{DISCUSSION}

Anemia and hematologic deficiencies may be etiological factors for the disease of RAS [7]. In a study of 12 patients found that $8 \%$ patient experienced RAS [2]. A number of hematologic deficiencies have been found to be more common in patients with RAS than in the general population. A study found that the overall frequency of hematologic deficiencies was $56.2 \%$ in 32 adult patients with RAS versus $7 \%$ of controls living in the same geographical area. Anemia, possibly caused by these deficiencies, was found in $34.4 \%$ of patients with RAS versus $6.9 \%$ of controls [5].

Multiple nutritional deficiencies including deficiencies of vitamins B1, B2, B6, and B12, folate, iron, and ferritin are considered to be the possible etiologies of RAS [9]. A recent study found that deficiencies of vitamin B12, folate, and iron, occurring alone or together, have been associated with aphthous stomatitis in patients of all ages [5]. Previous studies showed that 57 (20.9\%), 55 (20.1\%), $13(4.8 \%)$ and 7 $(2.6 \%)$ of 273 RAS patients have deficiencies of $\mathrm{Hb}$, iron, vitamin B12, and folic acid, respectively. Previous RAS study by Sun et al. described deficiencies of vitamins B1, B2, B6 and B12, folate, iron, ferritin and $\mathrm{Hb}$ in a portion of RAS patients. This study also showed that $\mathrm{Hb}$, serum iron, vitamin B12, and folic acid deficiencies can be detected in $20-43 \%, 19-36 \%$, 4$12 \%$, and $2-6 \%$ of RAS patients, respectively, depending on different subtypes of RAS patients [15].

IDA should be suspected in every case of RAS when no other obvious causes are identified. These findings are believed to be cause by the impaired cellular immunity, deficient bactericidal activity of polymorphonuclear leukocytes, inadequate antibody response, and epithelial abnormalities attributed to iron lack [2]. It is also believed that RAS patients with anemia and lower $\mathrm{Hb}$ levels have reduced capacity of the blood to carry oxygen to oral mucosa, finally resulting in atrophy of oral mucosa. In addition, iron is essential to the normal functioning of oral epithelial cells. Oral epithelial cells have a high turnover rate. Therefore, deficiencies of iron, vitamin B12, and folic acid may result in oral epithelial atrophy. Atrophic oral epithelium in hematinic-deficient patients may explain why some patients with hematologic deficiencies are prone to have RAS. Moreover, replacement therapy with hematinics for RAS patients with deficiencies of corresponding hematinics can result in a significant clinical improvement or at least a reduction in frequency and severity of their oral aphthous ulcers [7].

Previous study further explains why RAS itself may be an important factor causing anemia and hematinic deficiencies in RAS patients. The results of a previous diet history questionnaire study indicate a significantly lower daily intake of vitamin B12 and folate in minortyped RAS patients than in control subjects [16]. These results suggest that even the small oral ulcerations may impede the food intake by minor-typed RAS patients [7]. By definition, the major-typed RAS patients often have severer and larger recurrent oral ulcerations than minor-typed RAS patients [15]. These severer ulcerative lesions may cause burning sensation and pain of the lesional oral mucosa when the patients eat salty and spicy food stuffs. The eating difficulty may result in reduced food intake that in turn leads to anemia and hematinic deficiencies in a certain percentage of our RAS patients [7].

In conclusion, RAS is a multifactorial disease with one of the common suspected etiology is IDA. Hence routine hematological examination may be carried out in RAS patients.

\section{ACKNOWLEDGMENT}

The acknowledgments are addressed to The Research Institution (Lembaga Penelitian) of Universitas Sumatera Utara for funding this research by awarding the Talenta Grant 2017. The author would like to offer 
the outmost gratitude to this institution for fully supporting and funding of this review.

\section{REFERENCES}

[1] G.W. Mirowski. (2017, March 26) Aphthous stomatitis. Available: http://emedicine.medscape.com/article/1075570overview.

[2] S.B. Woo, M.S. Greenberg, Ulcerative, vesicular, and bullous lesions, and M.A. Huber, V.S. Sankar, Hematologic disease. In: Burket's oral medicine, $12^{\text {th }}$ ed., Connecticut: People's Medical Pubishing House, 2015, pp. 73-77, 440-441.

[3] L. Shruthi, S. Pushparaja, P. Bhavna, "Role of copper and iron deficiency in pathogenesis of recurrent aphthous ulcer," Int. Res. J. Pharm., vol. 4(5), pp. 219-211, May 2013.

[4] A. Altenburg, N. El-Haj, C. Micheli, M. Puttkammer, M.B. Abdel-Naser, C.C. Zouboulis, "The treatment of chronic recurrent oral aphthous ulcers," Dtsch. Arztebl., vol. 111, pp. 665-673, 2014

[5] N.R. Edgar, D. Saleh, R.A. Miller, "Recurrent apthous stomatitis: a review," J. Clin. Aesthet. Dermatol., vol. 10(3), pp. 26-36, March 2017.

[6] N.F. Khan, M. Saeed, A.A. Khan, "Correlation between hematological parameters and recurrent aphthous stomatitis," J. Pak. Dent. Assoc., vol. 19(2), pp. 124-128, 2010.

[7] H.P. Lin, Y.H. Wu, Y.P. Wang, Y.C. Wu, J.Y.F. Chang, A. Sun, "Anemia and hematinic dificiencies in anti-gasric parietal cell antibody-positive or all autoantibodies-negative recurrent aphtous stomatitis patients," Journal of the Formosan Medical Association, vol. 116, pp. 99-106, October 2016.
[8] M.A.O. Lewis, R.C.K. Jordan, A colour handbook oral medicine, $2^{\text {nd }}$ ed., London: Manson Publishing, 2012, pp. 26-27.

[9] Y.C. Wu, Y.H. Wu, Y.P. Wang, J.Y.F. Chang, H.M. Chen, A Sun, "Hematinic deficiencies and anemia statuses in recurren aphthous stomatitis patients with or without atrophic glossitis," journal of the Formosan Medical Association, vol. 115, pp. 1061-1068, October 2016.

[10] J.L. Harper. (2017, March 26) Iron deficiency anemia. Available: http://www.http://emedicine.medscape.com/article/202333overview\#a 1.

[11] J.E. Maakaron. (2017, March 26) Anemia. Available: http://www.http://emedicine.medscape.com/article/198475overview.

[12] B.K. Venkataraman, Diagnostic oral medicine. $1^{\text {st }}$ ed., New Delhi: Wolters Kluwer Health, 2013, pp. 649-653.

[13] A. Ghom, S. Ghom, Blood disorders. In: Textbook of ora medicine, $3^{\text {rd }}$ ed., New Delhi: Jaypee Brothers Medical Publishers (P) Ltd., 2014, pp. 858-859.

[14] A.G. Ghom, S.A. Ghom. Blood disorders. In: A.G. Ghom, S.A Ghom. Textbook of oral medicine. $3^{\text {rd }}$ ed. New Delhi: Jaypee Brothers Medical Publishers, 2014, pp. 858-859.

[15] A. Sun, H.M. Chen, S.J. Cheng, Y.P. Wang, J.Y.P. Chang, Y.C. $\mathrm{Wu}$, et al., "Significant association of deficiency of hemoglobin iron, vitamin B12, and folic acid and high homocysteine level with recurrent aphthous stomatitis. J Oral Pathol Med, vol. 44 pp. 300-305, 2015.

[16] S.T. Kozlak, S.J. Walsh, R.V. Lalla, "Reduced dietary intake of vitamin B12 and folate in patients with recurrent aphthous stomatitis,” J. Oral Pathol. Med., vol. 39, pp. 420-423, 2010 